\title{
DETERMINATION OF POLYCYCLIC AROMATIC HYDROCARBONS IN SOIL SAMPLES USING ULTRASONIC PROBE AND SALT-ASSISTED LIQUID-LIQUID EXTRACTION COUPLED WITH HIGH-PERFORMANCE LIQUID CHROMATOGRAPHY
}

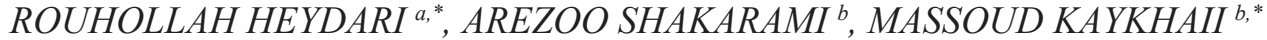 \\ ${ }^{a}$ Razi Herbal Medicines Research Center, Lorestan University of Medical Sciences, P.O. Box 68149-89468, Khorramabad, Iran \\ ${ }^{b}$ Department of Chemistry, Faculty of Sciences, University of Sistan and Baluchestan, Zahedan 98135-674, Iran
}

\begin{abstract}
Background: Polycyclic aromatic hydrocarbons (PAHs) are a class of chemicals which have 2 to 7 fused aromatic rings. It is demonstrated that even trace amounts of PAHs are carcinogens, mutagens, and teratogens which can lead to serious risk to the health of humans. According to these facts, determination of PAHs in environmental samples is essential.

Methods: In this study, ultrasonic in combination with salt-assisted liquid-liquid extraction was used efficiently for the extraction of PAHs from soil and water samples. In order to evaluate the performance of the proposed method three PAHs, naphthalene, anthracene and pyrene were selected as model analytes. Influential parameters on the extraction efficiency of analytes such as extraction solvent and its volume, salting-out agent and its concentration, ultrasonic time, ultrasonic amplitude and pulse were investigated and optimized.

Results: The optimum conditions were as follow; extracting solvent; tetrahydofuran, extracting solvent volume; $3 \mathrm{~mL}$, salting-out agent; sodium acetate, salting-out agent concentration; $20 \% \mathrm{w} / \mathrm{v}$, ultrasonic time; $10 \mathrm{~s}$, ultrasonic amplitude; $60 \%$ and ultrasonic pulse; $0.5 \mathrm{~s}$. The limits of quantitation for pyrene, naphthalene and anthracene were 1.0, 1.0 and $0.7 \mathrm{ng} \mathrm{g}^{-1}$, respectively. Under the optimum conditions, obtained recoveries in different matrices were in the range of 80.0 to $100.0 \%$ with a relative standard deviation better than $7.5 \%$.

Conclusions: In the proposed method, after the UAE, sample was exposed to SALLE without need of solid residue removal from the sample. Therefore, extraction steps such as filtration and centrifuge were removed which lead to time saving.
\end{abstract}

Keywords: polycyclic aromatic hydrocarbons; ultrasound; salt-assisted; liquid-liquid extraction; soil analysis

\section{INTRODUCTION}

Polycyclic aromatic hydrocarbons (PAHs) are a class of chemicals which have 2 to 7 fused aromatic rings. It is demonstrated that even trace amounts of PAHs are carcinogens, mutagens, and teratogens which can lead to serious risk to the health of humans [1]. These compounds are naturally present in coal, crude oil, and gasoline and released into the environment from both natural and anthropogenic sources. Also, PAHs are produced when coal, oil, gas, wood, garbage, and tobacco are burned. They are also highly mobile in the environment and can be easily distributed across air, soil, and water [2] According to these facts, determination of PAHs in environmental samples is essential; however, due to low concentration of these analytes and the severe matrix interferes in real samples such as soils, direct determination of them is challenging. Applying a separation and pre-concentration step before analysis can overcome this.

Solid-liquid extraction (SLE) methods allow to solve components of interest from solid samples using a suitable solvent. Various modes of SLE techniques such as maceration, ultrasonic-assisted extraction (UAE), microwave-assisted extraction (MAE), Soxhlet extraction (SE) and accelerated solvent extraction (ASE) were proposed for extraction from food, pharmaceutical, cosmetics and environmental samples [3-9]. Among these techniques, UAE is of high interest because of advantages such as low cost operation, simplicity and mild extraction conditions. This technique can be performed in two modes of ultrasonic bath and probe-type ultrasound devices which are based on a transducer as a source of ultrasound waves. Ultrasonic baths can be operating at thermostat mode with a frequency of around $40 \mathrm{kHz}$. They are cheap, available and large numbers of samples can be simultaneously treated. However, compared with probe types, the low reproducibility and power of ultrasound delivered to the sample are major disadvantages. Indeed, the delivered intensity is highly attenuated by the water contained in the bath and the container used for sample holding. That's why ultrasonic probes are generally preferred for extraction applications. Comparing to the ultrasonic bath, the probe system is more powerful due to an ultrasonic intensity delivered through an ultrasonic probe which is immersed directly into the sample container. They are generally operated at $\sim 20 \mathrm{kHz}$ and use transducer bonded to probe which is immersed into the reactor. This configuration results in a direct delivery of ultrasound to the extraction media with minimal ultrasonic energy loss [10].

Consumption of large amount of toxic and harmful organic solvents in classical SLE methods such as maceration and SE is a major challenge which cab be resolved by miniaturizing and coupling of extraction methods [11-15]. Two major disadvantages of SLE methods are using a large volume of extracting solvent which leads to analyte dilution and lack of selectivity for target compounds. These problems can be overcomed by coupling the SLE with other techniques such as liquid-liquid extraction (LLE) and solidphase extraction (SPE) methods. In this way, UAE can be coupled with the salt-assisted liquid-liquid extraction (SALLE) as a suitable LLE method for preconcentration of analytes.

The SALLE is an extraction method which can separate hydrophilic and hydrophobic compounds from the aqueous solution using a miscible organic solvent and an inert salt as extracting solvent and salting-out reagent, respectively [16-19]. This technique has advantages such as low cost, simplicity, high efficiency and it is compatible with a variety of detection systems [20].

In this study, ultrasonic in combination with salt-assisted liquid-liquid extraction was used efficiently for the extraction of PAHs from soil and water samples. Influential parameters on the extraction efficiencies of analytes were investigated and optimized. Finally, the analytical parameters of the proposed technique were compared with the published previously reports.

\section{MATERIALS AND METHODS}

\section{Chemicals and reagents}

Naphthalene, anthracene, pyrene, acetonitrile (ACN), tetrahydrofuran (THF), isopropyl alcohol (IPA), ammonium acetate $\left(\mathrm{NH}_{4} \mathrm{AOC}\right)$, sodium chloride $(\mathrm{NaCl})$ and sodium sulfate $\left(\mathrm{Na}_{2} \mathrm{SO}_{4}\right)$ were purchased from Merck KGaA Company (Darmstadt, Germany). All solutions were prepared using ultra-pure MilliQ ${ }^{\circledR}$ (Millipore, USA) purification system.

Chromatographic conditions

An HPLC system (Shimadzu Corporation, Kyoto, Japan) which consisted of a quaternary pump (LC-10ATvp), UV-Vis detector (SPD-M10Avp), vacuum degasser and system controller (SCL-10Avp) was used. A manual injector with a $10 \mu \mathrm{L}$ sample loop was applied for loading the sample. Class VP-LC workstation software was employed to acquire and process chromatographic data. A reversed-phase monolithic column (Chromolith ${ }^{\circledR}$ RP$18,100 \mathrm{~mm} \times 4.6 \mathrm{~mm}$ i.d., Merck KGaA, Germany) was used. The mobile phase was a mixture of water and acetonitrile $(80: 20, \mathrm{v} / \mathrm{v})$. Prior to preparation of the mobile phase, water and acetonitrile were degassed separately using a Millipore vacuum pump. The UV detector was set at $254 \mathrm{~nm}$. The flow rate and column temperature were adjusted at $1.0 \mathrm{~mL} \mathrm{~min}^{-1}$ and ambient temperature, respectively.

Real samples collection

Soil and water samples were collected from the areas near a gas station in the city of Khorramabad (Iran) and stored at $4{ }^{\circ} \mathrm{C}$. Samples were subjected to 
extraction without any pretreatment.

\section{Standard solutions}

Standard stock solutions were prepared by dissolving proper amounts of each analyte in methanol to make $100 \mu \mathrm{g} \mathrm{mL} \mathrm{m}^{-1}$ concentrations of them. Working standard solutions at different concentrations were prepared freshly by mixing the appropriate volumes of the stock solutions and diluting with deionized water.

Ultrasonic and salt-assisted liquid-liquid extraction

$1.0 \mathrm{~g}$ of spiking blank soil or $10 \mathrm{~mL}$ of aqueous sample was transferred to a centrifuge tube. Then, $10 \mathrm{~mL}$ deionized water, $2.6 \mathrm{~g}$ NaAOC and $3 \mathrm{~mL}$ THF were added to the tube and stirred for 1.0 minute. The mixture was subjected to ultrasonic probe. After ultrasonic, the mixture was allowed to phase separation and organic layer $(\approx 2 \mathrm{~mL})$ was withdrawn using a syringe and $10 \mu \mathrm{L}$ of it was injected to the HPLC system after filtering through a PTFE filter.

Blank soil was spiked with a mixture standard solution of three analytes at concentration of $50 \mathrm{ng} \mathrm{g}^{-1}$. Briefly, $1.0 \mathrm{~g}$ of blank soil was mixed with $0.5 \mathrm{~mL}$ of a mixture standard solution with concentration of $100 \mathrm{ng} \mathrm{mL}^{-1}$ for each analyte. Then, soil was dried in shadow and used as sample for method optimization.

\section{RESULTS AND DISCUSSION}

The extraction efficiency of analytes is affected by many parameters, such as type of extracting solvent, volume of extracting solvent, ultrasonic time, amplitude and pulse, type of salt and its concentration. The effects of these factors were studied and outlined below:

\section{Effect of extracting solvent and its volume}

Based on the previously published reports, common used solvents in SALLE method, such as ACN, THF, IPA and their mixtures were investigated $[17,18,21]$. Fig. 1 shows the effect of solvent type on the extraction efficiency of the analytes. As can be seen, the maximum extraction efficiencies were obtained using THF as extracting solvent. Fig. 2 shows the influence of extracting solvent volume on the extraction. It is observed the peak area of the analytes increased with increasing THF volume up to $3.0 \mathrm{~mL}$ and then decreased quickly. That's because at higher THF volumes, more organic solvent is dissolved in the aqueous phase which leads to increase the solubility of analytes in the aqueous phase, while applying more organic solvent increases the final volume of collected solvent and results in analyte dilution. Therfore, volume of $3.0 \mathrm{~mL}$ was selected as the optimum extracting solvent volume for further investigations.

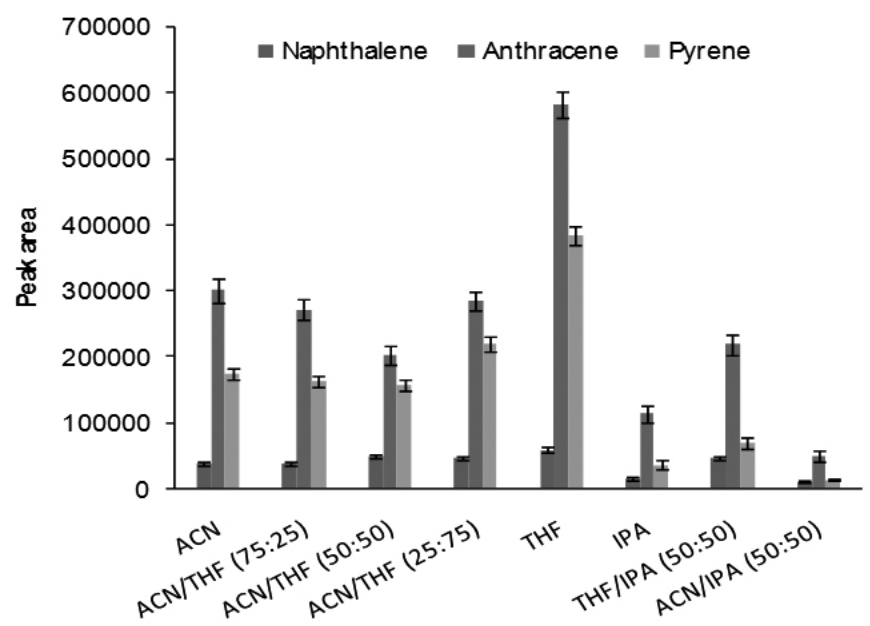

Fig. 1. Effect of extracting solvent on the extraction efficiencies. Extraction conditions; extracting solvent volume, $3 \mathrm{~mL}$; salt, $\mathrm{NaCl}$; salt amount, $20 \% \mathrm{w} / \mathrm{v}$; ultrasonic time, $5 \mathrm{~min}$; ultrasonic amplitude, $50 \%$; pulse duration, $0.5 \mathrm{~s}$.

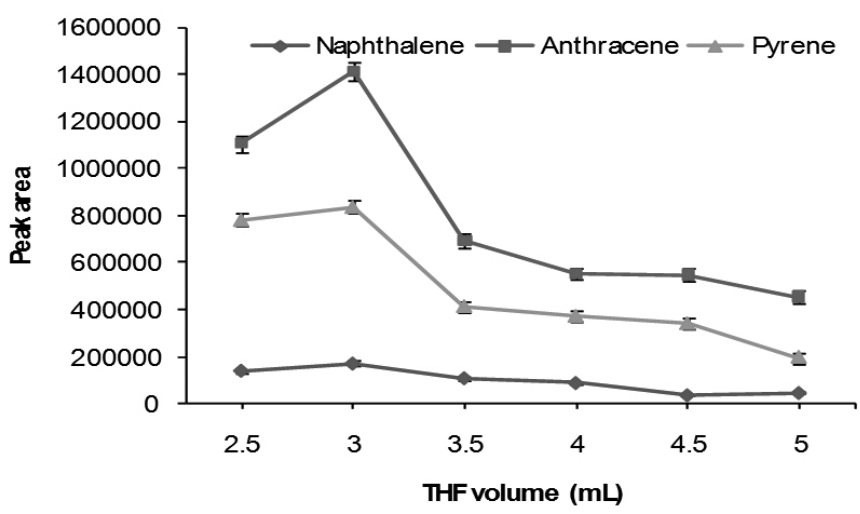

Fig. 2. Effect of extracting solvent volume on the extraction efficiencies. Extraction conditions; extracting solvent, THF; salt, NaCl; salt amount, 20 $\% \mathrm{w} / \mathrm{v}$; ultrasonic time, $5 \mathrm{~min}$; ultrasonic amplitude, $50 \%$; pulse duration, $0.5 \mathrm{~s}$.

\section{Effect of salt and its concentration}

The effects of several salts on the extraction efficiency of studied analytes were evaluated and results are shown in Fig. 3. As can be seen, $\mathrm{NaOAC}$ has the maximum salting-out effect in the proposed method.

The effect of salt concentration was investigated in the range of $15-55$ $\% \mathrm{w} / \mathrm{v}$. The results presented in Fig. 4 indicate that the extraction efficiency of analytes increases with the increase of salt concentration up to $20 \% \mathrm{w} / \mathrm{v}$ and then decreases. High salt concentration increases the viscosity of the aqueous phase which decreases the analyte mass transfer rate from the aqueous phase to organic phase. Therefore, $20 \% \mathrm{w} / \mathrm{v}$ was selected as the optimum salt concentration.

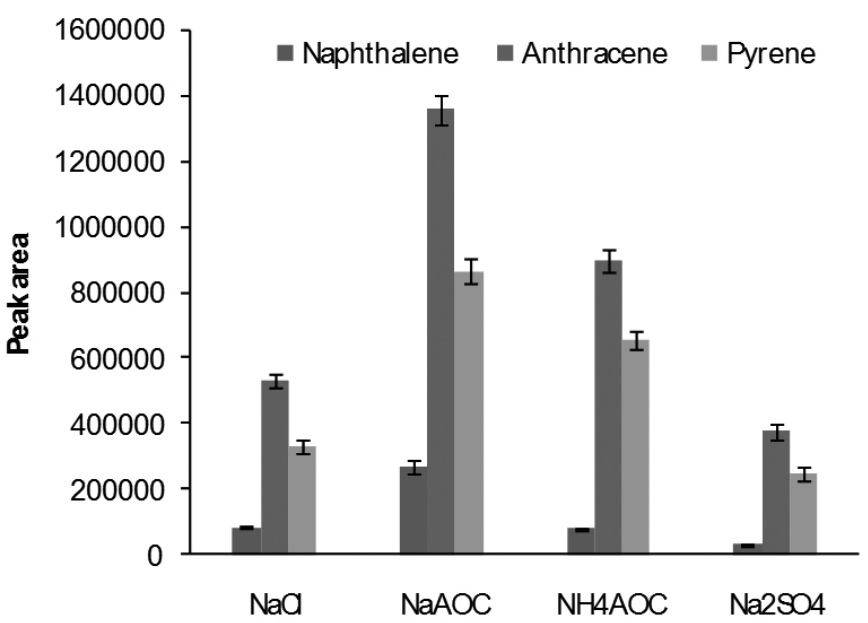

Figure 3. Effect of salt on the extraction efficiencies. Extraction conditions; extracting solvent, THF; extracting solvent volume, $3 \mathrm{~mL}$; salt amount, 20 $\% \mathrm{w} / \mathrm{v}$; ultrasonic time, $5 \mathrm{~min}$; ultrasonic amplitude, $50 \%$; pulse duration, $0.5 \mathrm{~s}$.

Effect of ultrasonic time, amplitude and pulse

Influence of ultrasonic time on extraction efficiency of analytes was investigated in the range of 5 to $210 \mathrm{~s}$. As can be seen in Fig. 5, increasing the ultrasonic time leads to increase of extraction efficiency up to $10 \mathrm{~s}$ and then the signal was decreased. At the beginning of process, increase of ultrasonic time can lead to analyte desorption from solid sample. Decreasing of extraction efficiencies at higher ultrasonic times $(>10 \mathrm{~s})$ can be attributed to analytes decomposition by ultrasonic waves. Consequently, $10 \mathrm{~s}$ was chosen as the optimum ultrasonic time in further experiments. 


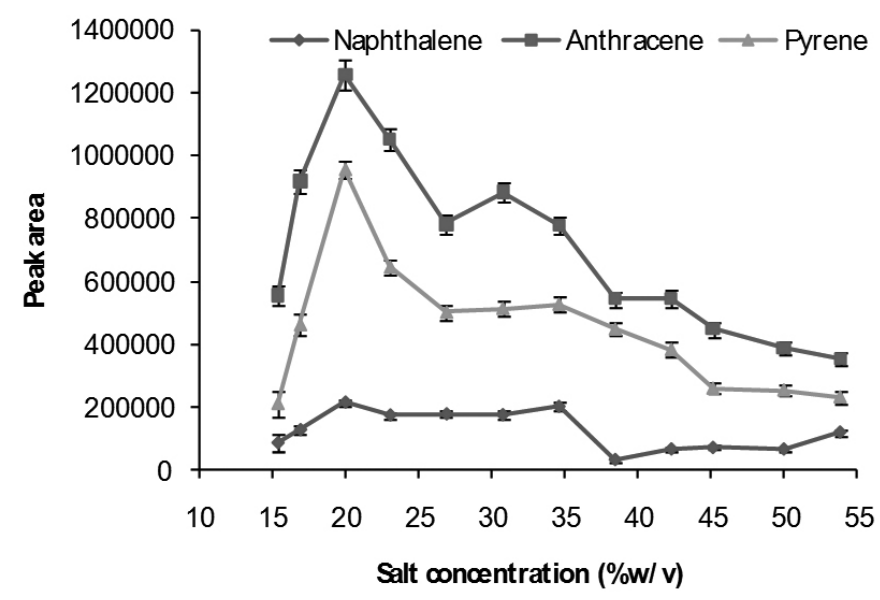

Figure 4. Effect of salt amount on the extraction efficiencies. Extraction conditions; extracting solvent, THF; extracting solvent volume, $3 \mathrm{~mL}$; salt, $\mathrm{NaAOC}$; ultrasonic time, $5 \mathrm{~min}$; ultrasonic amplitude, $50 \%$; pulse duration, $0.5 \mathrm{~s}$.

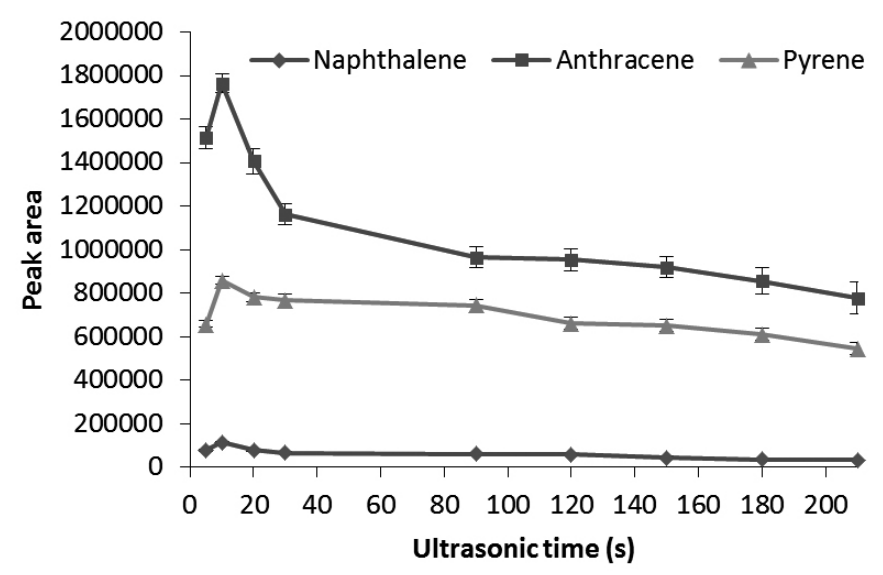

Figure 5. Effect of ultrasonic time on the extraction efficiencies. Extraction conditions; extracting solvent, THF; extracting solvent volume, 3 $\mathrm{mL}$; salt, NaAOC; salt amount, $20 \% \mathrm{w} / \mathrm{v}$; ultrasonic amplitude, $50 \%$; pulse duration, $0.5 \mathrm{~s}$.

The intensity of sonication is proportional to the amplitude of ultrasonic probe vibration. Therefore, higher amplitudes of vibrations lead to an increase in the intensity of vibrations and an increase in the analyte extraction from solid sample. However, the intensity of sonication can be adjusted using ultrasonic amplitude. The effect of ultrasonic amplitude on the extraction efficiencies of target analytes was investigated in the range of 20 to $100 \%$. Fig. 6 shows the effect of ultrasonic amplitude on the peak areas of analytes. As can be seen, 60 $\%$ can be selected as the optimum value.

Acoustic irradiation time in ultrasonic probe systems is adjustable using pulse mode. Pulse optimization was performed in the range of 0.1-1 s. The maximum extraction efficiency for all analytes was obtained at $0.5 \mathrm{~s}$ (Fig. 7). Therefore, $0.5 \mathrm{~s}$ were chosen as the optimum pulse duration in subsequent experiments.

Method validation

The figures of merit of the proposed method including linear dynamic ranges (LDRs), limits of detection (LODs), limits of quantitation (LOQs) and relative standard deviations (RSDs) for the determination of PAHs under the optimized conditions were determined (Table 1). The LODs and LOQs were calculated as concentrations of analytes which resulted in a chromatogram peak with a signal-to-noise (S/N) ratio of 3 and 10, respectively. Precision and accuracy data were obtained using spiked real samples with PAHs standard solutions in three concentration levels. Relative standard deviation (RSD) values for PAHs in three concentration levels were in the range of 4.9-7.5\% (Table 2). Relative recovery values were in the range of $80.0-100.0 \%$. The results in Table 1 and Table 2 indicate that this method can be successfully applied for the determination of studied PAHs in water and soil samples. Typical chromatograms of standard solution and extracted PAHs using the USALLE method are shown in Fig. 8. As can be seen, the proposed method can extract target analytes from the soil samples very efficiently.

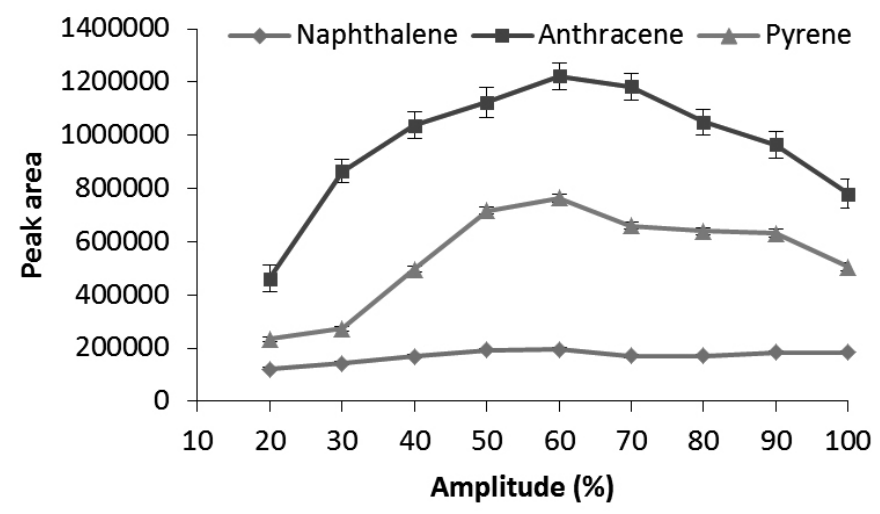

Figure 6. Effect of ultrasonic amplitude on the extraction efficiencies. Extraction conditions; extracting solvent, THF; extracting solvent volume, $3 \mathrm{~mL}$; salt, NaAOC; salt amount, $20 \% \mathrm{w} / \mathrm{v}$; ultrasonic time, $10 \mathrm{~min}$; pulse duration, $0.5 \mathrm{~s}$.

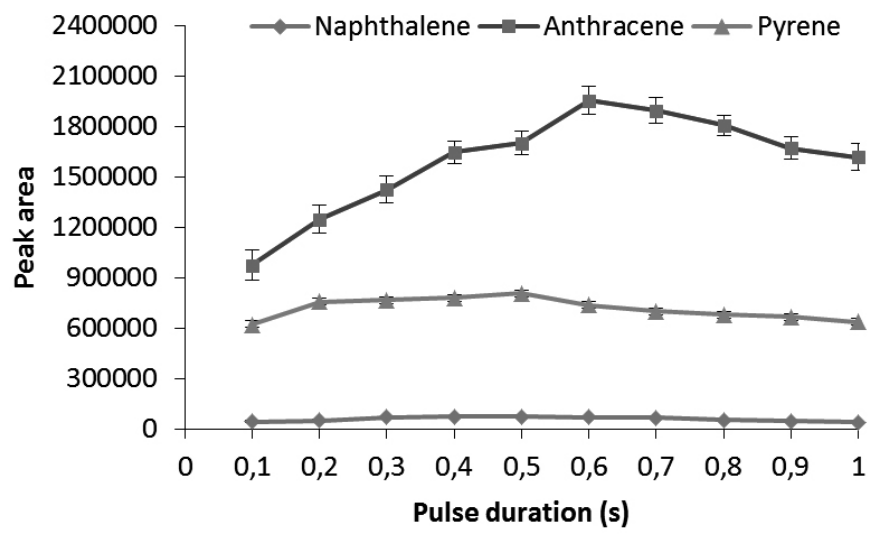

Figure 7. Effect of pulse duration on the extraction efficiencies. Extraction conditions; extracting solvent, THF; extracting solvent volume, $3 \mathrm{~mL}$; salt, NaAOC; salt amount, $20 \% \mathrm{w} / \mathrm{v}$; ultrasonic time, $10 \mathrm{~min}$; ultrasonic amplitude, $60 \%$;

Table 1. Some analytical parameters of the proposed method.

\begin{tabular}{|c|c|c|c|c|}
\hline Analyte & LDR $\left(\mathrm{ng} \mathrm{g}^{-1}\right)$ & $\mathrm{R}^{2}$ & LOD $\left(\mathrm{ng} \mathrm{g}^{-1}\right)$ & LOQ $\left(\mathrm{ng} \mathrm{g}^{-1}\right)$ \\
\hline Naphthalene & $1.0-200.0$ & 0.9973 & 0.3 & 1.0 \\
\hline Anthracene & $0.7-100.0$ & 0.9975 & 0.07 & 0.7 \\
\hline Pyrene & $1.0-200.0$ & 0.9971 & 0.3 & 1.0 \\
\hline
\end{tabular}


Table 2. Results of real sample analysis and recoveries tests.

\begin{tabular}{|c|c|c|c|c|c|c|}
\hline \multirow{2}{*}{ Samples } & \multirow{2}{*}{$\begin{array}{l}\text { Added }\left(n g^{-1}\right) \text { for } \\
\text { each analyte }\end{array}$} & \multicolumn{3}{|c|}{ Determined $\left(\mathrm{ng} \mathrm{g}^{-1}\right)$} & \multirow{2}{*}{ RSD (\%) (n=5) } & \multirow{2}{*}{ Recovery (\%) } \\
\hline & & Naphthalene & Anthracene & Pyrene & & \\
\hline \multirow{4}{*}{ Soil\#1 } & 0 & 48.0 & 23.0 & N.D. & \multirow{4}{*}{$5.0-7.2$} & \multirow{4}{*}{$90.0-95.0$} \\
\hline & 1 & 48.9 & 23.9 & 0.94 & & \\
\hline & 10 & 57.5 & 32.4 & 9.1 & & \\
\hline & 50 & 94.0 & 68.0 & 45 & & \\
\hline \multirow{4}{*}{ Soil\#2 } & 0 & 68.0 & 80.0 & 21.0 & \multirow{4}{*}{$4.9-7.5$} & \multirow{4}{*}{$80.0-91.0$} \\
\hline & 1 & 68.9 & 80.8 & 21.8 & & \\
\hline & 10 & 77.1 & 89.0 & 30.1 & & \\
\hline & 50 & 113.0 & 125.0 & 61.0 & & \\
\hline \multirow{4}{*}{ Soil\#3 } & 0 & $<\mathrm{LOD}$ & $<\mathrm{LOD}$ & 33.0 & \multirow{4}{*}{$4.9-7.3$} & \multirow{4}{*}{$84.0-93.0$} \\
\hline & 1 & 0.85 & 0.88 & 0.92 & & \\
\hline & 10 & 9.2 & 9.3 & 8.9 & & \\
\hline & 50 & 46.0 & 42.0 & 75.0 & & \\
\hline \multirow{4}{*}{ Water } & 0 & N.D. & N.D. & N.D. & \multirow{4}{*}{$5.1-7.5$} & \multirow{4}{*}{$84.0-100.0$} \\
\hline & 1 & 0.85 & 0.84 & 0.86 & & \\
\hline & 10 & 9.5 & 9.8 & 9.9 & & \\
\hline & 50 & 42.0 & 48.0 & 50.0 & & \\
\hline
\end{tabular}

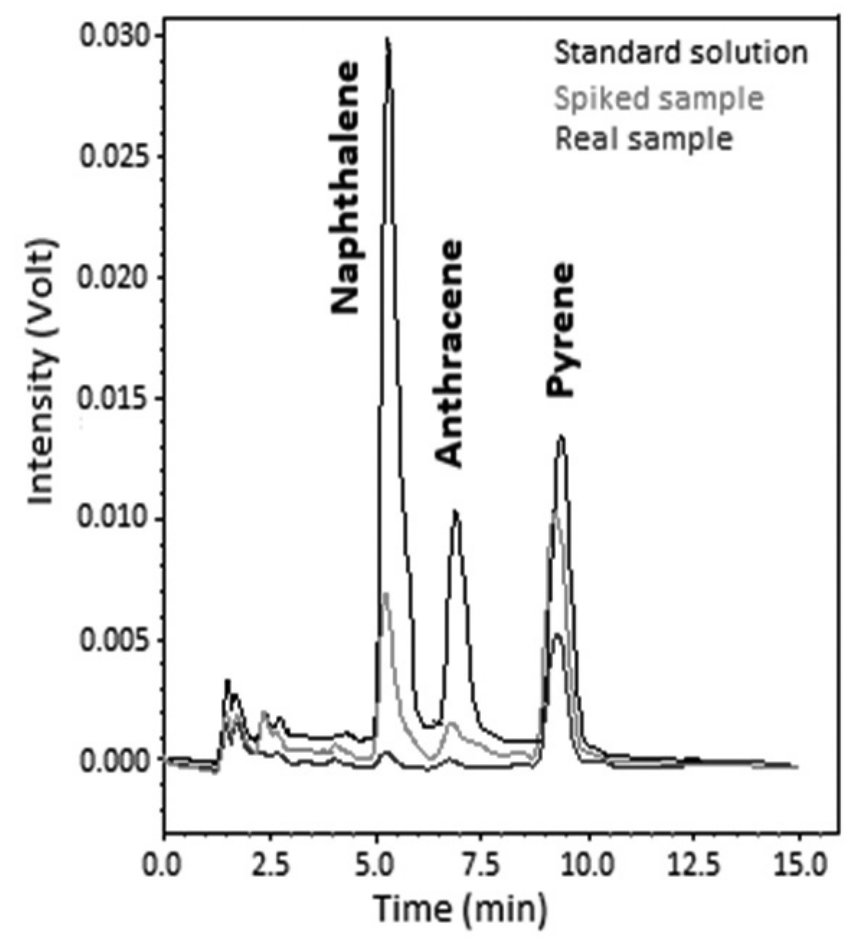

Figure 8. Typical chromatograms of standard solution of PAHs, spiked and unspiked soil samples using the proposed method under the optimized conditions. Concentration of analytes in standard solution were as follow: naphthalene; $200 \mathrm{ng} \mathrm{g}^{-1}$, anthracene $100 \mathrm{ng} \mathrm{g}^{-1}$ and pyrene $100 \mathrm{ng} \mathrm{g}^{-1}$.

Several analytical parameters of the proposed method were compared with previous reports in the literature which used SPE and SPME as sample preparation methods (Table 3). These techniques require more steps and used sorbent for preparation of SPE disks and SPME fibers are synthetic which lead to spend more time and cost. Moreover, most of those methods used mass detection systems which are not available in many laboratories. In one study, in an ultrasonic bath, PAHs were extracted from soil using cyclohexane as extracting solvent. Then, cyclohexane was evaporated in a rotary evaporator and residue was dissolved in methanol for injection to HPLC. In our proposed method, solvent evaporation and reconstitution steps are not necessary. As a final point, compare to other methods, analytical parameters of the proposed method such as LODs, LOQs, RSDs and recoveries are satisfactory. 
Table 3. Comparison of the analytical parameters of the proposed USALLE method with several published reports for the determination of PAHs from soil samples.

\begin{tabular}{|c|c|c|c|c|c|c|}
\hline Sample preparation technique & Analysis instrument & LOD (ng g $\left.{ }^{-1}\right)$ & LOQ $\left(\right.$ ng g $\left.^{-1}\right)$ & RSD (\%) & Recovery (\%) & Ref. \\
\hline NTD-SPME ${ }^{a}$ & GC-FID ${ }^{b}$ & $0.05-0.17$ & $0.2-0.6$ & $9.7-15.4$ & N.R. ${ }^{d}$ & {$[22]$} \\
\hline CA-INCAT-SPME ${ }^{c}$ & GC-FID & $0.002-0.02$ & $0.59-9.83$ & $7.7-11.0$ & N.R. & {$[23]$} \\
\hline $\mathrm{MSPE}^{\mathrm{e}}$ & GC-MS & $0.07-0.41$ & $0.24-1.37$ & $4.27-13.68$ & N.R. & {$[24]$} \\
\hline UAE & HPLC-FLU ${ }^{\mathrm{f}}$ & $0.02-0.44$ & N.R. & $2.0-15.0$ & $49.0-98.0$ & {$[25]$} \\
\hline CHaME-NTD $^{\mathrm{g}}$ & GC-FID & $0.005-0.038$ & N.R. & $6.2-9.8$ & $80.0-110.0$ & {$[26]$} \\
\hline
\end{tabular}

a Needle trap device-solid-phase microextraction

${ }^{\mathrm{b}}$ gas chromatography-flame ionization detector

${ }^{\mathrm{c}}$ Cooling-assisted-inside needle capillary adsorption trap

${ }^{\mathrm{d}}$ Not reported

${ }^{\mathrm{e}}$ Magnetic solid-phase extraction

${ }_{\mathrm{f}}^{\mathrm{f}}$ High-performance liquid chromatography- fluorometric detection

g cooling/heating-assisted microextraction needle trap device

\section{CONCLUSION}

In this study, UAE as a suitable SLE technique was coupled with SALLE for extraction, pre-concentration and clean-up of three PAHs from solid samples. The UAE process was applied using a probe. In the proposed method, after the UAE, sample was exposed to SALLE without need of solid residue removal from the sample. Therefore, extraction steps such as filtration and centrifuge were removed which lead to time saving. In addition, SALLE is a simple, cost-effective and fast technique which requires only a small volume of organic phase and has high compatibility with analysis system.

\section{CONFLICT OF INTEREST}

The authors declare no conflict of interest, financial or otherwise.

\section{ACKNOWLEDGEMENT}

Authors gratefully acknowledge the support of Razi Herbal Medicines Research Center, Lorestan University of Medical Sciences.

\section{REFERENCES}

1. C.E. Boström, P. Gerde, A. Hanberg, B. Jernström, C. Johansson, T. Kyrklund, A. Rannug, M. Törnqvist, K. Victorin, R. Westerholm, Environ. Health Perspect. 110, 451, (2002)

2. K.H. Kim, S.A. Jahan, E. Kabir, R.J.C. Brown, Environ. Int. 60, 71, (2013)

3. A. Ghassempour, R. Heydari, Z. Talebpour, A.R. Fakhari, A. Rassouli, N. Davies, H.Y. Aboul-Enein, J. Liq. Chromatogr. Relat. Technol. 31, 2686, (2008)

4. M. Hosseini, R. Heydari, M. Alimoradi, J. Sep. Sci. 38, 663, (2015)

5. R. Heydari, M. Hosseini, Chem. Nat. Compd. 53, 538, (2017)

6. A. Pourhossein, K. Alizadeh, J. Iran. Chem. Soc. 15, 303, (2018)

7. M.B. Gholivand, Y. Yamini, M. Dayeni, Y. Shokoohinia, J. Iran. Chem Soc. 12, 707, (2015)

8. R.K. Saini, Y.S. Keum, Food Chem. 240, 90, (2018)

9. K. Madej, T.K. Kalenik, W. Piekoszewski, Food Chem. 269, 527 (2018)

10. F. Chemat, N. Rombaut, A.G. Sicaire, A. Meullemiestre, A.S. FabianoTixier, M. Abert-Vian, Ultrason. Sonochem. 34, 540, (2017)

11. R. Heydari, Anal. Lett. 45, 1875, (2012)

12. M. Rashidipour, R. Heydari, A. Feizbakhsh, P. Hashemi, J. AOAC Int. 97, $1109,(2014)$

13. R. Heydari, S. Azizi, J. Chromatogr. Sci. 53, 1020, (2015)

14. M. Rashidipour, R. Heydari, A. Feizbakhsh, P. Hashemi, Nat. Prod. Res. 29, 621, (2015)

15. P. Salehi, A.R. Fakhari, S.N. Ebrahimi, R. Heydari, Flavour Fragr. J. 22 280, (2007)

16. R. Heydari, M. Hosseini, R. Rezaeepour, J. Iran. Chem. Soc. 14, 1691, (2017)

17. R. Rezaeepour, R. Heydari, A. Ismaili, Anal. Methods 7, 3253, (2015)

18. R. Heydari, S. Zarabi, Anal. Methods 6, 8469, (2014)
19. M. Hosseini, R. Heydari, M. Alimoradi, Talanta 130, 171, (2014)

20. Y.Q. Tang, N. Weng, Bioanalysis 5, 1583, (2013)

21. A. Ismaili, R. Heydari, R. Rezaeepour, J. Sep. Sci. 39, 405, (2016)

22. N. Heidari, A. Ghiasvand, S. Abdolhosseini, Anal. Chim. Acta 975, 11 (2017)

23. A.R. Ghiasvand, F. Yazdankhah, J. Chromatogr. A 1487, 47, (2017)

24. S.B. Qin, Y.H. Fan, X.X. Mou, X.S. Li, S.H. Qi, J. Chromatogr. A 1568 29, (2018)

25. M.N. Kayali-Sayadi, S. Rubio-Barroso, C.A. Díaz-Díaz, L.M. Polo-Díez, Fresenius J. Anal. Chem. 368, 697, (2000)

26. A.R. Ghiasvand, N. Heidari, S. Abdolhosseini, A. Hamdi, P.R. Haddad, Analyst 143, 2632, (2018) 